\title{
Construction of Optimum Wavelet Packets for Multi-Carrier Based Spectrum Pooling Systems
}

\author{
M. K. Lakshmanan • H. Nikookar
}

Published online: 4 April 2009

(C) The Author(s) 2009. This article is published with open access at Springerlink.com

\begin{abstract}
The growing popularity of wireless applications has placed enormous burden on valuable resources such as spectral bandwidth. This has brought about a major revamp of traditional resource allocation policies culminating in an explosion of research activity in the field of cognitive radio (CR) (Mitola, Cognitive radio: an integrated agent architecture for software defined radio, Doctoral dissertation, 2000; Haykin, J Sel Areas Commun 23:201-220, 2005). In this paper we demonstrate the operation of a Wavelet Packet based multi-carrier modulation (WP-MCM) scheme in the context of cognitive radio. The wavelet packet (WP) bases are derived from multistage tree-structured paraunitary filter banks. To enable the WP-MCM cognitive radio system to co-exist with other licensed users a common spectrum pool is maintained and the WP-MCM transmission waveform characteristics are shaped to communicate in the idle time-frequency gaps of the licensed user. This is achieved by dynamically deactivating wavelet packet carriers in and near the region of the licensed user spectrum. Various wavelets including the well-known families Daubechies, Coiflet, Symlet are applied and studied. The emphasis is on the design and development of optimal WP carriers that have narrow and well confined spectral footprints. To this end filter banks that are maximally frequency selective are derived through a modified Remez exchange algorithm. Through simulation results the ability of the proposed wavelet packet based mechanism in seamlessly cohabiting with licensed users is demonstrated. The Bit Error rate (BER) performance is shown to be comparable, and even at times better, to the conventional Fourier based OFDM system.
\end{abstract}

Keywords Wavelet packets · Multi-carrier modulation ·

Maximally frequency selective wavelets $\cdot$ Spectrum pooling

M. K. Lakshmanan $(\varangle) \cdot$ H. Nikookar

Department of Electrical Engineering, Mathematics and Computer Science, International Research Center for Telecommunications and Radar (IRCTR), Delft University of Technology, Mekelweg 4, 2628 CD Delft, The Netherlands

e-mail: m.k.lakshmanan@tudelft.nl

H. Nikookar

e-mail: h.nikookar@tudelft.nl 


\section{Introduction}

\subsection{Background}

Wireless interoperable communication networks are now a reality. They have spawned many new and exciting applications like mobile entertainment, mobile internet access, healthcare and medical monitoring services, data sensing in sensor networks, smart homes, combat radios, disaster management, automated highways and factories. With each passing day newer and newer such services are being launched even while existing services continue to flourish. And demand for wireless services is likely to continue for the foreseeable future. However, with increasing popularity of the wireless services the demands on prime resources like radio spectrum are put to great test. For example the Federal Communications Commission (FCC), which handles frequency allocations in the United States, has already assigned most radio spectrum to various services and does not have enough frequency bands to cater to new services or to expand existing ones.

Even when radio spectrum appears to be completely choked, an FCC study conducted in 2002 revealed that much of the available spectrum is underused most of the time (only $20 \%$ or less of the spectrum is used) and that spectrum congestions are more due to the sub-optimal use of spectrum than to the lack of free spectrum [6]. The paradox of non-availability of spectrum even when large portions of licensed spectrum is underutilized most of the time has prompted a rethinking in existing spectrum regulatory policies. While traditional spectrum allocation schemes follow a static approach where established frequency bands are allocated and assigned to fixed licensees, the new approach envisioned is a Dynamic Spectrum Access (DSA) model where unlicensed users may rent unused spectrum from licensed users on a need-to-need basis. To actualize this vision the development of wireless systems that intelligently adapt their transmission parameters (including spectrum occupied) in accordance with the changing environment and requirements to maximize resource utilization has been promoted. Cognitive radio [14] is an attempt in that direction. It is an advanced technology for the efficient use of radio resources. It learns from the environment and adaptively changes the parameters of radio transmission including modulation scheme, transmit power, carrier frequency, channel coding to ensure highly reliable communication $[14,6]$. Further, it can discover under-utilized spectrum by spectrum sensing and can adapt its transmission settings accordingly without causing interference to existing/legacy users.

Existing literature recommends the use of Spectrum Pooling, a novel spectrum management protocol, for enhancement of spectrum utilization. Spectrum pooling enables public access to spectral ranges of licensed frequency bands which are seldom used by overlaying a secondary rental user (RU) to an existing licensed user (LU). The LUs are radio systems authenticated to operate under licensed spectral bands. The RUs are intelligent CR systems that actively scan the landscape and opportunistically utilize available and unused resources. The RU relinquishes control over the resources (here spectrum) as and when the LU starts using them. To identify and utilize unused bands, the frequency bands of various radio systems (including licensed and rental users) are combined to obtain a common spectral pool. The transmission waveform of the RU is shaped in a way that it utilizes the unoccupied timefrequency gaps of the LU paving way for LU and RU systems coexistence. The pioneering work on the subject was conducted by Weiss and Jondral [22] who devised a spectrum pooling scheme using multi-carrier modulation (MCM) where individual sub-carriers adjacent to the occupied licensed user spectrum are vacated for frequencies which are occupied by a licensed user. The combination of adaptive MCM and spectrum pooling has been found 
to be a robust method to achieve a good quality of communication and efficient use of the spectrum.

\subsection{Theme of Article-Wavelet Packet Based Multicarrier Modulation for Spectrum Pooling systems}

In traditional implementations of MCM, as in Orthogonal Frequency Division Multiplexing (OFDM), the generation and modulation of the sub-channels is accomplished digitally using Fourier bases. In this work we propose replacing the conventional Fourier-based complex exponential carriers of OFDM with orthonormal wavelet packet bases for use in cognitive radio environments $[7,12,15,1]$. The wavelet packet bases are derived from perfect reconstruction two-band FIR filter bank solutions [5,20,21,13,4,19,3]. Cohabitation of the WP-MCM based CR systems with existing licensed users is actualized by shaping its transmission waveform by adaptively activating or deactivating sub-carriers in a way that it utilizes the unoccupied time-frequency gaps of the LU. The idea is to dynamically sculpt the CR transmission signal so that it has no or very little time-frequency components competing with the LU. This way the CR can seamlessly blend with the LU operation. The steps to generate the WP-MCM transmission signal are elucidated in Fig. 1.

The key thrust is in the design and development of wavelets suitable for the application in hand. In theory any function that is essentially both time-limited and frequency-limited can be used. However in practice, a chosen set of wavelets have to satisfy a number of requirements. The wavelet bases cannot therefore be arbitrarily chosen and instead a general framework that accounts for the requirements in hand have to be defined and the wavelet of choice be selected through a principled approach. In this article we provide introductory material to design maximally frequency selective wavelet packet bases that best suit applicability towards spectrum pooling MCM systems.

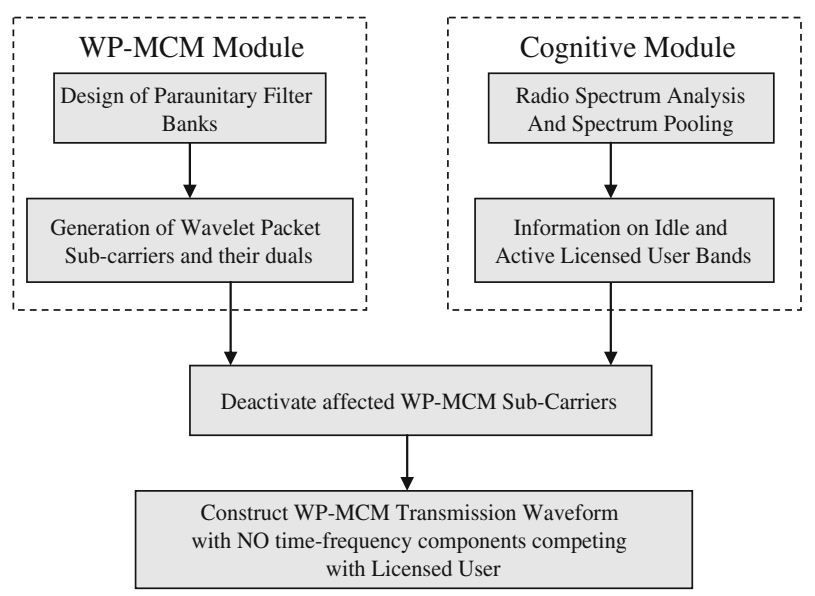

Fig. 1 Fundamental blocks of WP-MCM based cognitive radio system 


\subsection{Organization of Article}

The paper is organized as follows. We start by highlighting a few benefits of using wavelets for cognitive systems in Sect.2. The major elements of the proposed system are listed in Sect. 3. Section 3 will also detail the implementation of a wavelet packet based Multi-carrier modulation scheme. Section 4 will discuss the procedure to design the best wavelet bases for the WP-MCM based CR system. The simulation results are discussed in Sect.5. The inferences are drawn in Sect. 6.

\section{Motivation for Wavelet Packet Based Multicarrier Modulation Scheme}

Several reasons motivate applicability of wavelets based MCM to cognitive radio. Here we list few important ones [7] and [9]:

\subsection{Semi-Arbitrary Division of the Signal Space and Multirate Systems}

Wavelet transform can create subcarriers of different bandwidth and symbol length. Since each subcarrier has the same time-frequency plane area, an increase (or decrease) of bandwidth is bound to a decrease (or increase) of subcarrier symbol length. Such characteristics of the wavelets can be exploited to create a multirate system. From a communication perspective, such a feature is favorable for systems that must support multiple data streams with different transport delay requirements.

\subsection{Flexibility with Time-Frequency Tiling}

Another advantage of wavelets lies in their ability to arrange the time-frequency tiling in a manner that minimizes the channel disturbances. By flexibly aligning the time-frequency tiling, the effect of noise and interference on the signal can be minimized. Wavelet based systems are capable of overcoming known channel disturbances at the transmitter, rather than waiting to deal with them at the receiver. Thus, they can enhance the quality of service (QoS) of wireless systems.

\subsection{Sensitivity to Channel Effects}

The performance of a modulation scheme depends on the set of waveforms that the carriers use. The wavelet scheme therefore holds the promise of reducing the sensitivity of the system to harmful channel effects like Inter-symbol interference (ISI) and Inter-carrier interference (ICI).

\subsection{Flexibility with Sub-Carriers}

The derivation of wavelets is directly related to the iterative nature of the wavelet transform. The wavelet transform allows for a configurable transform size and hence a configurable number of carriers. This facility can be used, for instance, to reconfigure a transceiver according to a given communication protocol; the transform size could be selected according to the channel impulse response characteristics, computational complexity or link quality. 


\section{Sytem Blocks and Characteristics}

\subsection{Operation of WP-MCM Based Cognitive Radio System}

The system model of the proposed setup is illustrated in Fig. 2. The major blocks on the transmitter are the spectrum estimator and discrete inverse wavelet packet (IDWPT) modulator. And at the receiver the main component is the discrete wavelet packet transformer

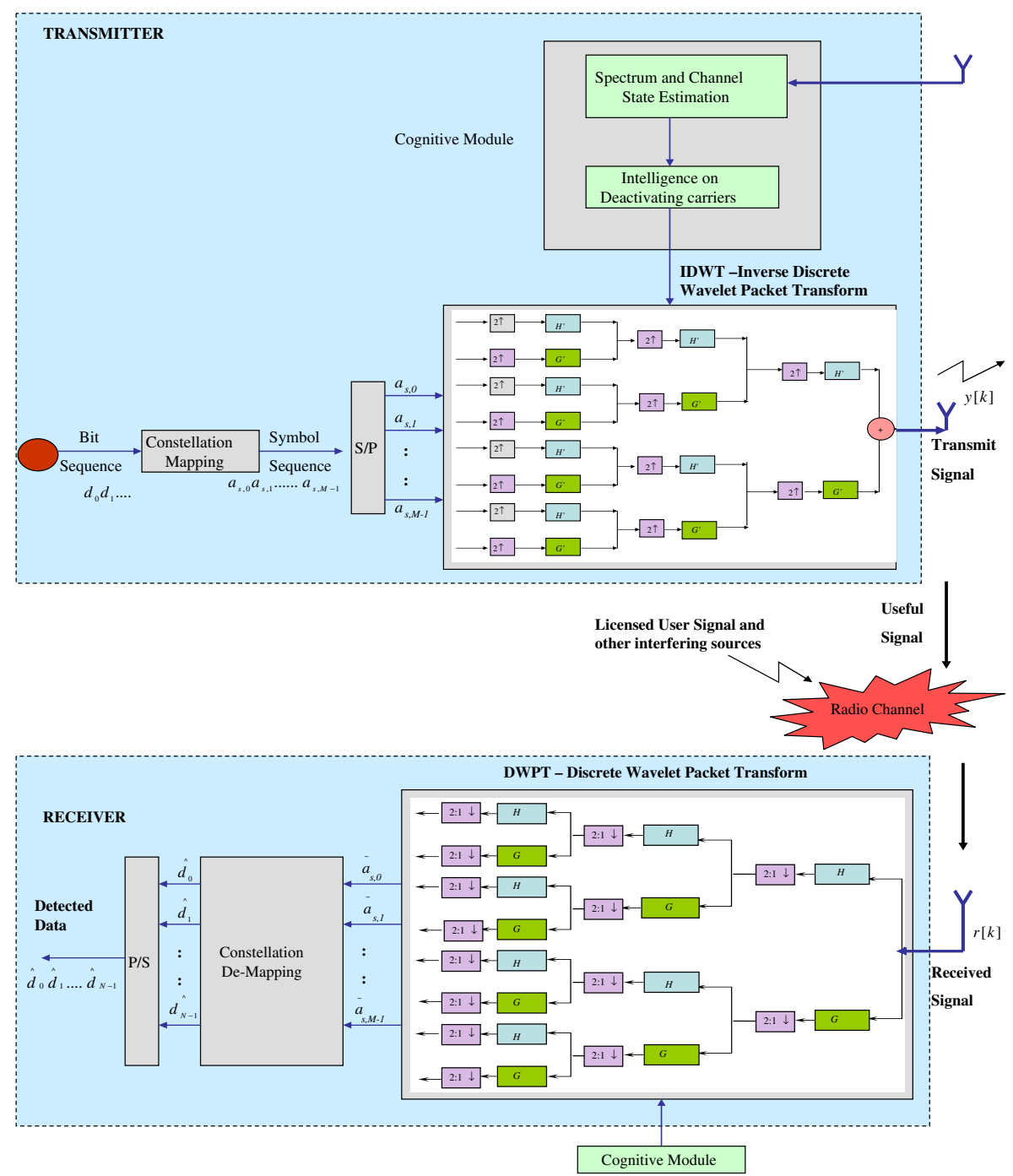

Fig. 2 Functional block diagram of the proposed system. The major blocks in the transceiver include the radio spectrum estimator and the inverse Wavelet packet transformer. And at the receiver the main component is the wavelet packet transformer. IDWPT and DWPT is implemented using filter bank analysis (to be explained later) with a set of 4 filters. $H^{\prime}$ and $G^{\prime}$ are the low and high pass reconstruction filters while $H$ and $G$ are the low and high pass decomposition filters. Down arrow depicts down-sampling and up arrow refers to up-sampling 
(DWPT). The chief goal of the system is wavelet packet based multicarrier modulation and transmission waveform shaping to enable seamless cohabitation with a licensed user.

WP-MCM is implemented by utilization of IDWPT at the transmitter and DWPT at the receiver, analogous to IDFT and DFT in OFDM systems. At the transmission end, an incoming high-rate serial data stream is split into $M$ lower-rate parallel streams. The data in each parallel branch is then up-sampled by $M$ and used to modulate $M$ subcarriers. Meanwhile, the spectrum estimator gauges the channel and performs a radio scene analysis to estimate LU frequency bands and detect spectrum holes. Based on the spectrum estimates, the cognitive modules dynamically vacate those sub-channels of the WP-MCM system that lie in and around the spectrum of the LU The idea is to dynamically sculpt the transmission signal of the RU in a way it has no or very little time-frequency components competing with the LU. This way the CR will appear invisible to the LU and seamlessly blend with its operation. The sub-carriers are then modulated and scaled to the desired energy level to obtain the WP-MCM transmission signal.

The transmitted signal in the discrete domain, $y[k]$, is composed of successive modulated symbols, each of which is constructed as the sum of $M$ sub-carriers, $\psi_{m}[k]$, individually modulated. It can be expressed in the discrete domain as:

$$
y[k]=\sum_{s} \sum_{m=0}^{M-1}\left(a_{s, m} \psi_{m}[k-s M]\right)
$$

where $a_{s, m}$ is a constellation encoded $s$ th data symbol modulating the $m$ th waveform. To reduce probability of error, the sub-carriers are made orthogonal. The orthogonality of the subcarriers is given as follows:

$$
<\psi_{m}[k], \psi_{n}[k]>=\delta[m-n]
$$

where <.,.$>$ represents the scalar product operator and $\delta$ is the kronecker delta with $\delta[i]=1$ if $i=0$, and is 0 otherwise.

In OFDM, the discrete functions $\psi_{m}[k]$ are the complex basis functions $p[k], e^{\frac{j 2 \pi m k}{M}}$ limited in the time domain by the window function $p[k]$ [7]. In the WP-MCM scheme, the sub-carrier waveforms are obtained through filter bank analysis by successively decomposing a couple of elementary paraunitary filter banks $[5,20,21,13,4,19,3]$.

In the receiver DWPT is performed to bring the signals back to their original domain. In one iteration of DWPT the input signal is filtered by high pass filter (HPF) and Low pass filter (LPF), decomposing original signal into two parts. Each of the decomposed parts is then downsampled by two satisfying the Nyquist rule. The receiver sub-carrier waveforms $\widetilde{\psi_{m}}[k]$ are the duals of $\psi_{m}[k]$ and their orthogonality can be expressed as:

$$
<\psi_{m}[k], \tilde{\psi_{n}}[k]>=\delta[m-n]
$$

The estimate of each subchannel $\hat{a}_{s, m}$ is given as:

$$
\hat{a}_{s, m}=(r[k] * \tilde{\psi m}[k-s M]) \downarrow_{M}
$$

where $r[k]$ is the received signal, * denotes the convolution operator and the down arrow represents decimation by $M$.

These estimates are then de-mapped and parallel to serial converted to identify the transmitted data. The transmitter and receiver are kept cognizant at all times on the nature of 


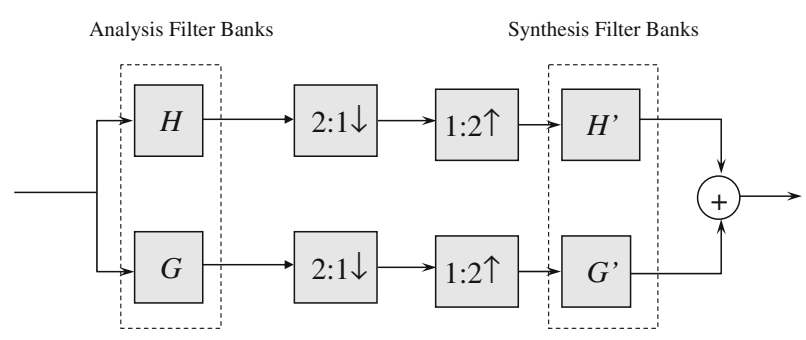

Fig. 3 Two channel filter bank analysis with analysis filters $H$ and $G$ (low and high pass, respectively) and synthesis filters $H^{\prime}$ and $G^{\prime}$ (low and high pass, respectively)

channel state and the transmission signal by correspondence of information through a pilot channel. ${ }^{1}$

The nature and characteristics of the filters used in IDWPT and DWPT greatly influence the WPM carries derived. By changing the parameters of the filter design the carrier-characteristics can be customised to any requirement. WPM thus gives designers a lot of freedom that can be used to design case-characteristic systems. More material on this subject is provided in the next section.

\subsection{Generation of Wavelet Packet Bases and Their Duals Through Two Channel Filter Bank Analysis}

\subsubsection{Background}

WP-MCM transmission is realized by replacing orthonormal complex exponential basis functions, as used in OFDM systems, with orthonormal wavelet packet basis functions. The wavelet packet bases and their dual bases are derived from multistage tree-structures derived by cascading 2-channel perfect reconstruction filter banks of the form shown in Fig. 3.

Multiresolution analysis of wavelet theory allows us to represent wavelet and scaling functions by HPF and LPF, respectively. Therefore the wavelet transformation can be easily implemented using discrete time filters. In this work we derive the carriers of the multicarrier system through DWPT. The DWPT is just like the wavelet transform except that it decomposes even the high frequency bands, which are kept intact in the wavelet transform. The coefficients of wavelet packet filter banks are computed using iterations of two channel filter bank decomposition or reconstruction. Wavelet packets are defined recursively by

$$
\begin{aligned}
& \psi_{k+1,2 p-1}[m]=\sqrt{2} \sum_{m^{\prime}} h\left[m^{\prime}\right] \psi_{k, p}\left[m-2^{k} m^{\prime}\right] \\
& \psi_{k+1,2 p}[m]=\sqrt{2} \sum_{m^{\prime}} g\left[m^{\prime}\right] \psi_{k, p}\left[m-2^{k} m^{\prime}\right]
\end{aligned}
$$

In (5) $\psi_{k, p}[m]$ denotes $p$ th wavelet packet function at the $k$ th level and $h[m]$ and $g[m]$ represent the impulse responses of the low and high pass filters, respectively.

\footnotetext{
${ }^{1}$ Here we assume that the intelligence to gauge the channel state is available only at the transmitter end. The transmission cycle will be suitably altered should the feature to measure radio spectrum be available at the receiver end too.
} 


\subsubsection{Generation of Wavelet Packet Sub-Carrier Bases}

The wavelet packet sub-carriers (to be used at the transmitter end) are generated through a multichannel filterbank consisting of cascaded two-channel filters applying the synthesis filters $\left(H^{\prime}\right.$ and $\left.G^{\prime}\right)$. This represents an inverse discrete wavelet packet transformation or IDWPT and consists of binary interpolation (up-sampling) by 2, filtering and recombination at each level. Figure 4a illustrates the derivation of 8 wavelet packet bases from a cascaded level-3 tree structure. The number of iterations $J$ determines the number of subcarriers $M$ generated and the relationship is given as $M \leq 2^{J}$. The time domain representation of the wavelet packet bases $\psi_{i}[k]$ is obtained through a simple convolution rule as given in (6).

$$
\begin{aligned}
& \psi_{i}[k]=f(k) * f(k / 2) * \cdots * f\left(k / 2^{J-2}\right) * f\left(k / 2^{J-1}\right) ; \\
& \text { where, } 0 \leq i \leq 2^{J}-1 \\
& \text { and, } f(k)= \begin{cases}h^{\prime}(k), & \text { for lowpass branches } \\
g^{\prime}(k), & \text { for highpass branches }\end{cases}
\end{aligned}
$$

Here $h^{\prime}$ and $g^{\prime}$ stand for the impulse responses of the low and high pass synthesis filters, respectively.

a
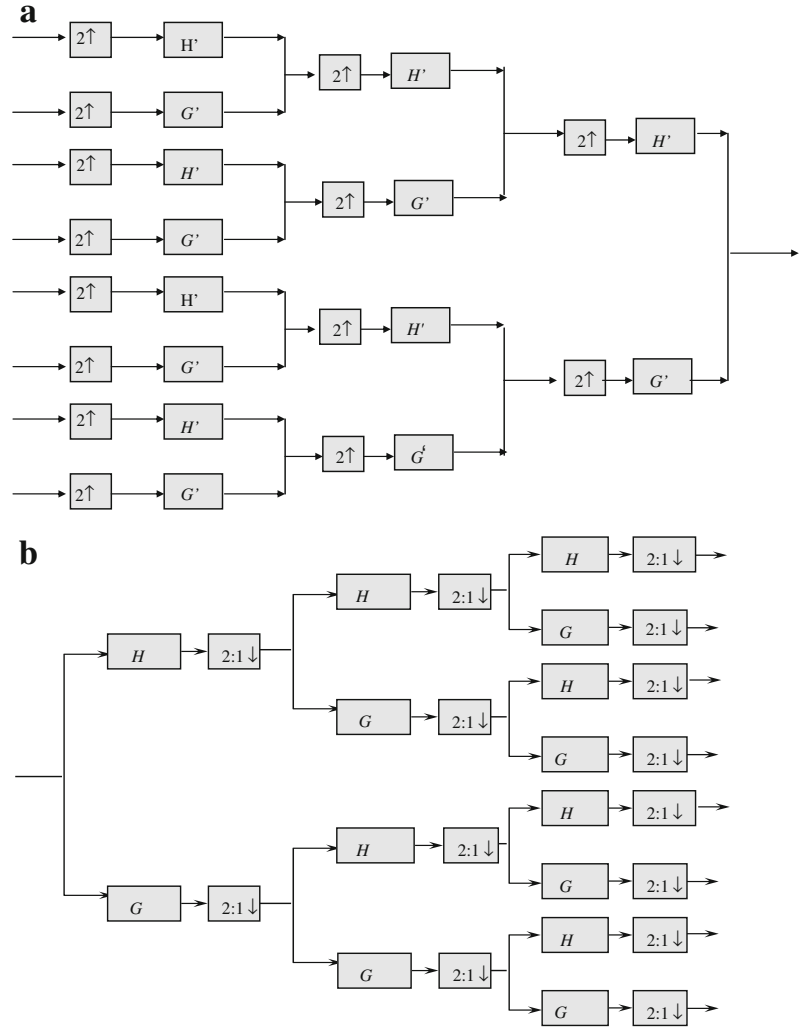

Fig. 4 a Generation of wavelets. A level-3 tree gives 8 wavelet packet bases. The up arrows represent interpolation by $2 . H^{\prime}$ and $G^{\prime}$ denote the frequency responses of the low and high pass reconstruction filters, respectively, $\mathbf{b}$ generation of wavelet Duals. A level-3 tree gives 8 wavelet packet dual bases. The down arrows represent decimation by $2 . H$ and $G$ denote the frequency responses of the low and high pass decomposition filters, respectively 


\subsubsection{Generation of Wavelet Packet Dual Bases}

The wavelet packet duals (to be used at the receiver end) are obtained from multichannel filter bank analysis too, though the processes are reversed. The duals are obtained from the analysis filters $(H$ and $G)$ through the analysis procedure which consists of filtering, decimation (down sampling) by 2 and decomposition at each stage. This process is called discrete wavelet packet transformation or DWPT. First the signal is passed through a half-band high and low pass filter. The half-band low pass filter removes all frequencies that are above half of the highest frequency, while the half-band high pass filter removes all frequencies that are below half of the highest frequency of the signal. Such a half-band filtering halves the resolution, but leaves the scale unchanged. The signal is then sub-sampled by two since half of the number of samples is redundant, according to the Nyquist's rule. This decomposition halves the time resolution since only half the number of samples then comes to characterize the entire signal. Conversely, it doubles the frequency resolution, since the frequency band of the signal spans only half the previous frequency band effectively reducing the uncertainty by half. This procedure is iteratively repeated till the desired degree of resolution. Figure $4 \mathrm{~b}$ illustrates the generation of 8 wavelet packet duals from a level-3 tree cascaded filter bank.

The duals $\widetilde{\psi}_{i}[k]$ are derived as:

$$
\begin{aligned}
& \widetilde{\psi}_{i}[k]=f(k) * f(2 k) * \cdots * f\left(2^{J-2} k\right) * f\left(2^{J-1} k\right) ; \\
& \text { where, } 0 \leq i \leq 2^{J}-1 \\
& \text { and, } f(k)= \begin{cases}h(k), & \text { for lowpass branches } \\
g(k), & \text { for highpass branches }\end{cases}
\end{aligned}
$$

In the Eq. $7 h$ and $g$ denote the impulse responses of the low and high pass analysis filters, respectively. And Fig. 5 shows the wavelet packet sub-band pulses derived from a 3 -stage cascading of maximally frequency selective wavelets (with specifications length $L=50$, regularity $K=19$ and transition band $B=0.1$ ) and their corresponding 8-channel spectrum. More on the frequency selective wavelets is explained in the next section.

\subsection{Identification and Removal of Affected Carriers}

In order to isolate and remove the carriers that are in the neighborhood of the LU, it is important to understand their frequency ordering. The frequency ordering of wavelet packet carriers is not in a sequence bur rather in binary Gray code order. This is because the output of any 2-channel analysis is the result of low/high pass filtering followed by down sampling. Down sampling generates two new filter results with half the number of elements. In addition to this it also results in mirroring of the high pass components. This switches the order of low and high pass components in a subsequent decomposition. When the wavelet packet algorithm is recursively applied the frequency ordering of the resultant carriers follow the Gray code sequence [8].

\section{Design of Best Wavelet Packet Bases For WP-MCM}

\subsection{Wavelet Theory}

The attributes of a multicarrier modulation system greatly depends on the set of waveforms it uses. The property of the waveforms in turn is determined by the underlying wavelet bases 
Fig. 5 Wavelet packet bases as MCM carriers (eight carriers). a Sub-band pulses in time domain, b spectra of carriers. The wavelet packet bases are derived from maximally frequency selective (described in Sect.4) paraunitary filter banks with specifications $L=50, K=19, B=0.1$
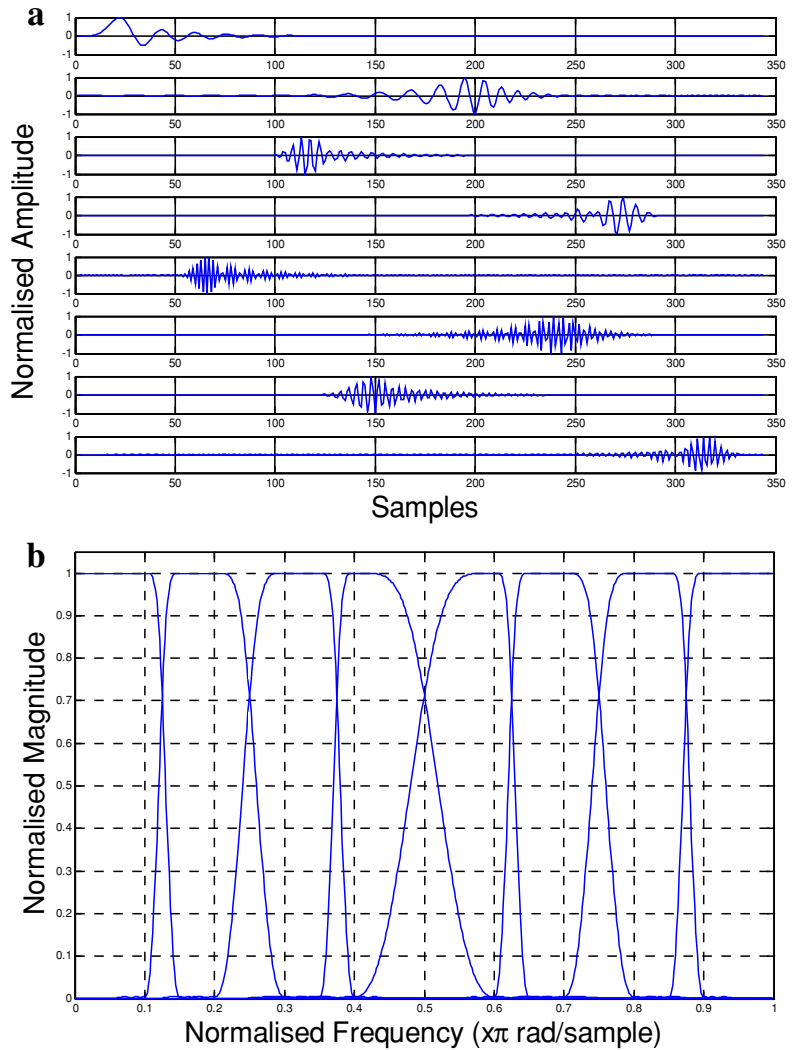

used. Many considerations go into the design of a wavelet system including properties such as orthogonality, compact support, symmetry, and smoothness. Here we shall discuss a few important ones.

\subsubsection{Paraunitary Condition}

The paraunitary condition is essential for many reasons. Firstly, it is a prerequisite for generating orthonormal wavelets [20]. Second, it automatically ensures perfect reconstruction of the decomposed signal [20] i.e., the original signal can be reconstructed without amplitude or phase or aliasing distortion, if the filter banks used satisfy the paraunitary condition. A rational transfer function $A(z)$ is said to be paraunitary when it obeys the relation $\widetilde{A}(z) A(z)=1$. Here $\tilde{A}(z)$ is the paraconjugate of $A(z)$ and is given as $\tilde{A}(z)=A^{\dagger}\left(z^{-1}\right)$ where the superscript $\dagger$ denotes the conjugation of the coefficients.

Only paraunitary filters are considered in this article and for such solution pairs the high pass and low pass filters share the relationship [5,20,21]:

$$
g[L-1-n]=(-1)^{n} h[n]
$$


where $L$ is the length of the filters. Further, paraunitary filters automatically satisfy the perfect reconstruction criterion [7] with the decomposition and reconstruction filters being complex conjugate time reversed versions of one another i.e.

$$
h^{\prime}[n]=h^{\dagger}[-n] \text { and } g^{\prime}[n]=g^{\dagger}[-n]
$$

Filters satisfying this condition are commonly used in signal processing, and are known as the Quadrature Mirror Filters (QMF). A nice import of these relations is that it is enough to design a single filter, either the low or high pass filter alone.

\subsubsection{Compact Support}

This property ensures that the wavelet is of finite duration and the filter banks used to derive the wavelets has a finite number of non-vanishing coefficients [3].

Properties 4.1.1 and 4.1.2 are necessary and sufficient conditions for the wavelets to be realized. However, they may not always guarantee the generation of smooth, regular and well shaped wavelets. Quite often the wavelets can be irregular or even fractal shaped [22]. Therefore to ensure smoothness or regularity of the wavelets the additional property of regularity is defined.

\subsubsection{Regularity}

This property is a measure of smoothness of the wavelet. The regularity condition requires that the wavelet be locally smooth and concentrated in both the time and frequency domains. It is normally quantified by the number of times a wavelet is continuously differentiable. The simplest regularity condition is the "flatness" constraint which is stated on the low pass filter. A LPF is said to satisfy $K$ th order flatness if its transfer function $H(z)$ contains $K$ zeroes located at the Nyquist frequency $(z=-1$ or $\omega=\pi)$. Parameter $K$ is called the regularity order and for a filter of length $L$ it satisfies the relation $0 \leq K \leq L / 2$.

Wavelets are defined by the wavelet function $\psi(t)$ (i.e. the mother wavelet) and scaling function $\phi(t)$ (also called father wavelet) in the time domain. Another way to determine the regularity of the wavelets is in terms of the number of vanishing moments of the wavelet and scaling functions [3] and used the dual vanishing moments to determine the convergence rate of the multiresolution projections. The $j$ th moments of the wavelet and scaling functions, $m_{w}(j)$ and $m_{s}(j)$, respectively, are defined in continuous time domain as follows:

$$
m_{w}(j)=\int t^{j} \psi(t) d t \text { and } m_{s}(j)=\int t^{j} \varphi(t) d t
$$

\subsection{Wavelet Families}

In this work we shall largely deal with the Daubechies family and its variants.

\subsubsection{Daubechies}

The Daubechies are a family of orthonormal wavelets with compact support with highest degree of smoothness. It was derived by Ingrid Daubechies [5] who used all the degrees of freedom $K$ to generate a wavelet family of maximum regularity for a given filter length $L$, or minimum $L$ for a given regularity [3]. This she did by imposing the maximum number of zero moments to the wavelet function in the vanishing moments' condition (Eq. 10). 


\subsubsection{Coiflet}

Coiflets are a variation of the Daubechies wavelets. They are so named because it was derived by Daubechies at the behest of R. Coifman who suggested the construction of orthonormal wavelet basis with vanishing moment conditions for both wavelet and scaling functions (unlike Daubechies where only the wavelet functions have zero moments). The wavelet function has $2 L$ moments equal to 0 and the scaling function has $2 L-1$ moments equal to 0 .

\subsubsection{Symlet}

The symlet family of wavelets is another variant of the Daubechies family which are nearlysymmetrical (as opposed to being symmetrical). These modifications were also proposed by Daubechies and the properties of the two wavelet families are similar.

\subsection{Choosing the Right Wavelet}

Choosing the right wavelet is a delicate and at times even an overwhelming task. In theory any time and frequency limited function can be utilized. However in practice, the wavelet bases cannot be arbitrarily chosen and instead have to satisfy a number of requirements. In general the choices to make can be with regard to the system of representation (continuous or discrete), properties of the wavelets desired (orthogonality/biorthogonality, regularity/smoothness, frequency selectivity), the application in hand and the context of use [2]. A framework that accounts for these requirements must first be defined and the wavelet selected in a principled approach through optimization of the wavelet design parameters.

\subsection{Wavelet Design Considerations for WP-MCM application}

With regard to the applicability to WP-MCM systems, the desirable properties may be listed as follows:

- The wavelet bases must be time-limited

- The bases must be well confined in frequency.

- The wavelet packet bases and their duals must be orthogonal (or at least linearly independent) to one another to enable perfect reconstruction.

- The bases must be orthogonal (or at least linearly independent) to one another in order to have unique demodulation.

- The bases must enable the system to handle channel effects and other distortions.

- The system must be easily realizable and must permit application of fast algorithms.

And in the filter bank domain the objective of the design procedure translates to construction of filters with the characteristics that they:

- have finite impulse response (FIR)

- are maximally frequency selective

- allow orthonormal expansion and perfect reconstruction of discrete-time signals

- satisfy the paraunitary condition

- satisfy a desired flatness/regularity condition 


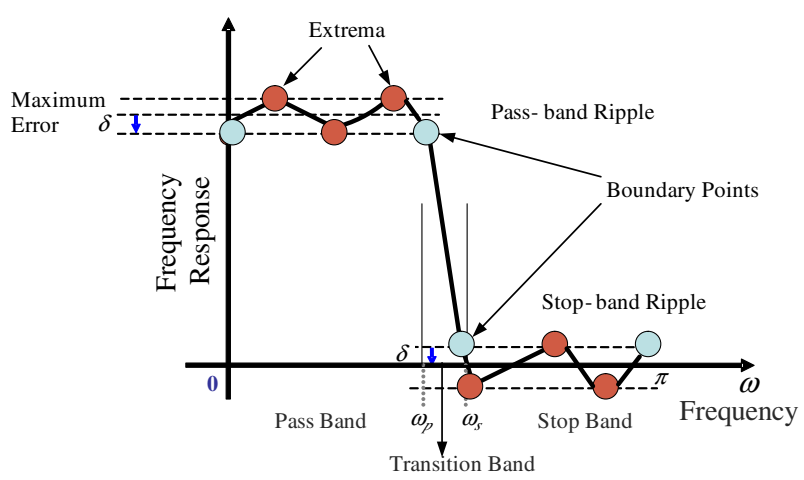

Fig. 6 Filter characteristics in frequency. In the figure $\omega_{p}$ and $\omega_{s}$, denote pass and stop band frequencies, respectively, $\left[0, \omega_{p}\right]$ is called the pass-band, $\left[\omega_{s}, \pi\right]$ is called the stop-band and $\left[\omega_{p}, \omega_{s}\right]$ is the transition band. $\delta$ is the tolerance or ripple

Amongst these properties the paraunitary and regularity properties are mandatory to the design of the filter banks. In addition to these properties the criterion that needs specific focus is the property of frequency selectivity.

\subsection{Need for Maximally Frequency Selective Filter Banks}

In an ideal scenario the filter banks used to generate the wavelets have zero transition bands $B$, i.e., difference between pass and stop band frequencies (refer Fig. 6). Under such an ideal scenario the wavelet packet bases derived from a level- $i$ decomposition have confined spectral footprints with bandwidth $\left(1 / 2^{i}\right)$ times that of the Nyquist frequency. However, available wavelet families are derived from filter banks that have a wide transition band and hence the resultant wavelet sub-carriers have a dispersed spectrum with footprints spilling into neighboring regions. The wider the transition bandwidth the greater the dispersion of the carrier's spectral footprint and therefore the greater the difficulty in isolating those sub-carriers that fall in the region of the licensed user. This greatly reduces the efficiency of the system. It is therefore important to design filter banks that have narrow transition bands.

\subsection{Design of Maximally Frequency Selective Filter Banks}

The design procedure comprises of defining a low pass FIR filter, satisfying the regularity, paraunitary and frequency selectivity conditions, expressed in the form of an impulse response $h(n)$ or a transfer function $H(z)$ or a difference equation. For a filter of length $L$ this is essentially solving $L$ unknown filter coefficients from $L$ linear equations. Of these $L$ linear equations, $L / 2$ equations come from the paraunitary constraint, $K$ equations come from the regularity or flatness constraint and the remaining $L / 2-K$ conditions offer the room for maneuverability to establish the desired wavelet property such as frequency selectivity. The larger the value of $L / 2-K$, the greater the degree of freedom for frequency selectivity and the greater the loss in regularity. There is therefore a trade-off between frequency selectivity and regularity. Wavelets such as the Daubechies family are maximally flat with regularity order $K=L / 2$ and hence they are not frequency selective.

To implement the frequency selective filters, the design parameters are stated in the frequency domain in terms of the desired magnitude response of the LPF as shown in Fig. 6. 
In the figure $\omega_{p}$ and $\omega_{s}$, denote pass and stop band frequencies, respectively, $\left[0, \omega_{p}\right]$ is called the pass-band, $\left[\omega_{s}, \pi\right]$ is called the stop-band and $\left[\omega_{p}, \omega_{s}\right]$ is the transition band. $\delta$ is the maximum value of the tolerance or ripple. The design goal is to generate the filter with a desired transition band even while the maximum error $\delta$ in the pass/stop-band is minimized.

The fundamental theory on the design of frequency selective filter banks was developed by Rioul and Duhamel $[17,18]$. They devised the procedure to design maximally frequency selective filter banks under a given set of constraints using the Remez exchange algorithm. The Remez exchange algorithm is an optimization algorithm that is commonly used in the design of FIR filters. It is popular because of its flexibility and computational efficiency. Also known as the Parks-McClellan algorithm, it works by converting the filter design problem into a problem of polynomial approximation [16]. The algorithm is an application of the Chebyshev alternation theorem that constructs the polynomial of best approximation to a desired function under a set of constraints. Through a minimax approximation the scheme seeks to arrive at a $l$ th order approximation polynomial function $C(x)$ that best approximates a desired filter polynomial function $B(x)$ (in our case the LPF $H(z)$ that satisfies the design specifications) in the given interval such that the absolute maximum error is minimized. The error is defined here as the weighted difference between the desired filter polynomial function and the approximation polynomial function and is given as

$$
E(x)=W(x)(B(x)-C(x))
$$

$E(x)$ and $W(x)$ are respectively the error and weighting polynomial functions. All polynomial functions are of the form $\sum_{i=0}^{l} p_{i} x^{i}$, with coefficients $p_{i}$ and degree of the polynomial function $l$. Chebyshev proved that such a polynomial $C(x)$ exists and that it inique. He also gave the criteria for a polynomial to be a minimax polynomial. The algorithm states that in the interval of consideration, the necessary and sufficient condition that $C(x)$ is the unique mini-max polynomial solution of degree $l$ is that there are at least $(l+2)$ points at which the error function $E(x)$ attains the absolute maximum value $\delta$ with alternating sign i.e.

$$
E\left(x_{i}\right)=-E\left(x_{i+1}\right)= \pm \max _{x \in I}\{|E(x)|\}=(-1)^{i} \delta
$$

for $x_{1}<x_{2}<\cdots<x_{l+2}$ in the desired interval $I$. Parks and McClellan proved that this approach could be used to derive a filter of a given length with minimal ripple. The right set of extremal points $x_{i}$ is arrived through an iterative procedure. In each iteration an interpolation problem is solved and the reference set of extremal points is updated. Rioul and Duhamel [17] deduced that $L / 2-K+1$ extremal points in the pass-band are necessary and sufficient to characterise a unique and optimal solution.

The procedure starts by choosing an arbitrary set of $L / 2-K+1$ points in the given interval. These $L / 2-K+1$ points help form $L / 2-K+1$ linear equations. The filter coefficients are obtained by solving the $L / 2-K+1$ linear equations in a way that the error at the $L / 2-K+1$ points considered is equal in magnitude and alternating in sign. It cannot be guaranteed after the first step that solution satisfies the minimax condition for the error function. That is the magnitude of the error need not be the absolute maximum magnitude in the interval of consideration. In order to find the minimax solution, the second step of the algorithm seeks new set of $L / 2-K+1$ points that approach the $L / 2-K+1$ points of the minimax solution. The new set of is determined by locating those points where the slope of the error function $E(x)$ is zero. Once these points are identified, the old set of $L / 2-K+1$ points is exchanged with the new points. This process is iteratively performed till the desired set of points that satisfy the minimax solution is obtained. The algorithm is said to have converged when the set of extremal points remains unchanged. Once the right set of extremal 


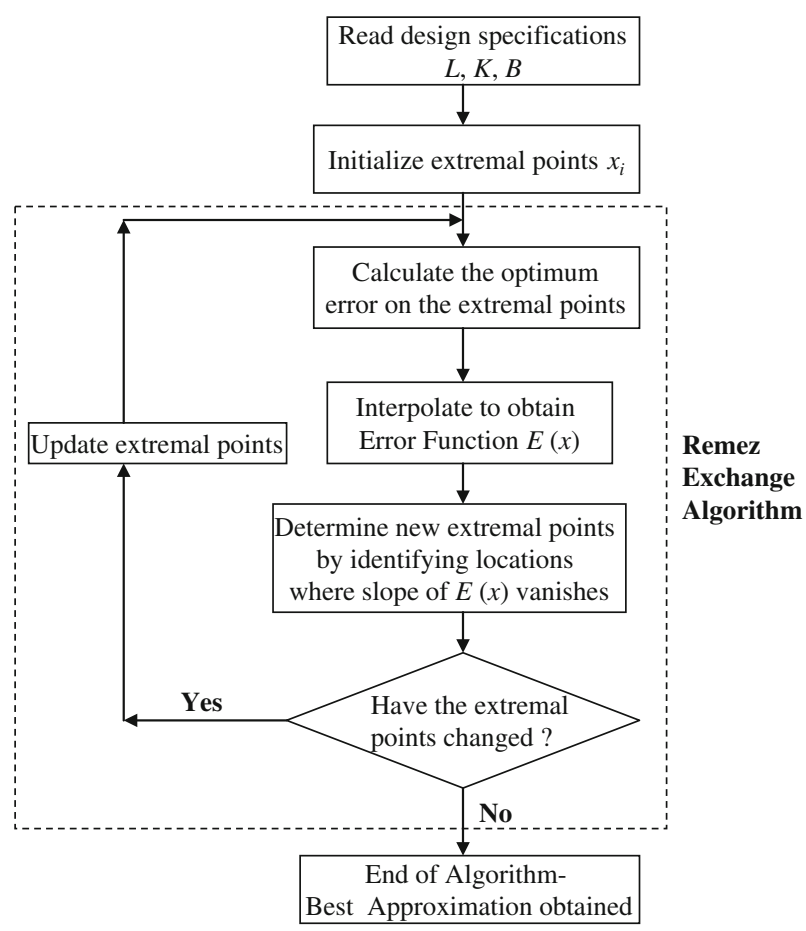

Fig. 7 Flowchart of the Remez exchange algorithm

points is identified, the optimum error and the filter can be obtained. The exact details of how these equations are solved to obtain the low pass filter $H(z)$ using the modified Remez exchange algorithm can be found in [17,18]. A flow chart of the algorithm is shown in Fig. 7. From the low pass filter $H(z)$, the high pass filter $G(z)$ and the reconstruction filters $\left(H^{\prime}(z)\right.$ and $\left.G^{\prime}(z)\right)$ can be obtained by applying (8) and (9).

\section{Simulation Results}

We now present a few computer simulation results that demonstrate the working of the WP-MCM based CR system.

\subsection{Simulation Setup—Characteristics of Cognitive Radio and Licensed User Systems}

To illustrate the operation we consider a setup where the CR system operates with 128 wavelet packet bases. The LU considered in this article is a wideband OFDM system with a bandwidth of about 1/4th of the CR system (comparable to 32 carriers of CR system) and is taken to be located in the middle of CR system (refer to Fig. 8). Both the CR and LU systems have the same transmission power. The 128 wavelet packet carriers are derived from a level-7 cascaded tree. To improve the performance of the CR, those sub-carriers that are in the region of the LU spectrum are removed. 


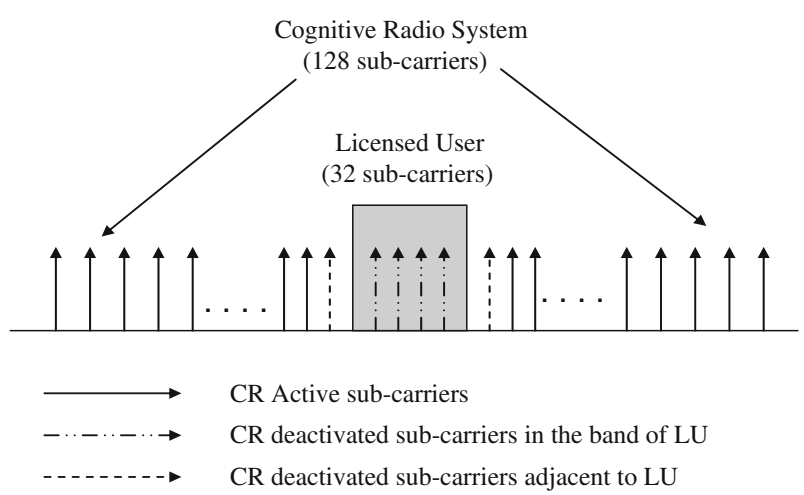

Fig. 8 Cognitive Radio (CR) and Licensed User (LU) characteristics

To simplify the evaluation of the system performance, the channel environment is assumed to be Additive White Gaussian Noise (AWGN) with no multi-path effects or fading (and hence no Inter Symbol Interference (ISI)). Due to perfect synchronization in AWGN channels, distortions such as Inter Carrier Interference (ICI) are therefore not considered and the radio transmission signal sequence is taken to be composed of pulse waveforms that do not overlap in time. Both the CR and LU systems apply quadrature phase shift keying (QPSK) as the modulation scheme of choice. Lastly, the cognitive modules at the transmitter and receiver are taken to be aware of the transmission signal characteristics including information on the nature of LU and the carriers that have to be active/deactivated at all times.

\subsection{Choice of Maximally Frequency Selective Filter Parameters (L, K and B) for Best System Performance}

As seen in the previous section the nature of the maximally frequency selective filter banks is influenced by three design parameters, namely, the length of the filter $L$, the regularity order $K$, and the transition bandwidth $B$. We will first see how these three parameters affect the wavelet packet bases and their duals, and hence the system operation.

\subsubsection{Influence of Regularity/Flatness Index $K$}

Two aspects of regularity order affect the system:

1. Frequency selectivity: with decreasing $K$ the filter characteristics become more and more frequency selective. This results in narrower transition bands and a better confinement of the spectral footprints of the resultant carriers.

2. Loss of smoothness: however, decreasing $K$ also results in loss of regularity (i.e. the derived carriers will have rough edges) which could lead to a loss in performance.

\subsubsection{Influence of Transition Bandwidth B}

With regard to the best choice of $B$, there are two limiting factors too, namely, the narrowness of transition band and presence of ripples in pass/stop bands. The narrower the transition bandwidth the better the performance. However, the reduction of the bandwidth 
is accompanied by ripples in the pass/stop bands which may worsen system performance. Hence, there is a limit to which the transition bandwidth can be reduced.

\subsubsection{Influence of Filter Length L}

The length of filter has a direct impact on the system performance. Longer the filter length greater the possibility to maneuver the other filter parameters, $K$ and $B$, to arrive at the optimal combination. However, it is important that the filter length is kept within practically realizable limits.

A better coverage on the influence of $L, K$, and $B$ on the system performance can be found in $[10,11]$.

The key is therefore to identify the best combination of $L, K$ and $B$ such that the transition band is less and the ripples are tolerable. During the simulation studies we considered a set of various $L, K$ and $B$ permutations to arrive at the optimum combination. The ranges of $L$ were between 20 and 50; $B$ was varied from 0.05 and 0.15 while $K$ was in the region $L / 2-6$ to $L / 2$. From the set considered the best performance was obtained for the combination $L=50, K=19, B=0.1$. In the following simulations we shall use these specifications to derive the filter banks and wavelet packets.

\subsection{Performance of WP-MCM Based CR System}

\subsubsection{Performance of WP-MCM Based Cognitive Radio System in the Presence of Licensed User}

Figure 9a plots the BER performance curves of the WP-MCM based CR system in the scenarios: absence of LU (only AWGN), presence of LU without any carrier deactivation and presence of LU with carrier deactivation. From the plots it is quite clear that the presence of $\mathrm{LU}$ affects the CR performance. And when the CR transmission is communicated around the $\mathrm{LU}$ with carriers in and around the region of interference removed, the CR system recuperates. Best results are obtained when 40 or more of the CR carriers in and around the region of the LU spectrum are removed. And when 44 carriers are removed seamless co-existence with LU is possible with performance almost matching the AWGN case (when LU is not present).

\subsubsection{Performance of Licensed User in the Presence of WP-MCM Based Cognitive Radio System}

It is highly imperative that the CR access to free/idle bands of LU does not cause any deleterious effects on the LU communication. To gauge this effect we present in Fig. $9 \mathrm{~b}$ the performance of the LU system under CR.

The relevant BER curves are for operation of the LU under the conditions: absence of CR (AWGN channel), presence of CR without any carrier deactivation and presence of CR with carrier deactivation. As in the case above, the deactivation of CR carriers is indeed necessary to prevent any loss in LU performance. For the scenario considered in this work, a noticeable improvement in the performances is obtained when 40 or more of the CR carriers are removed. 

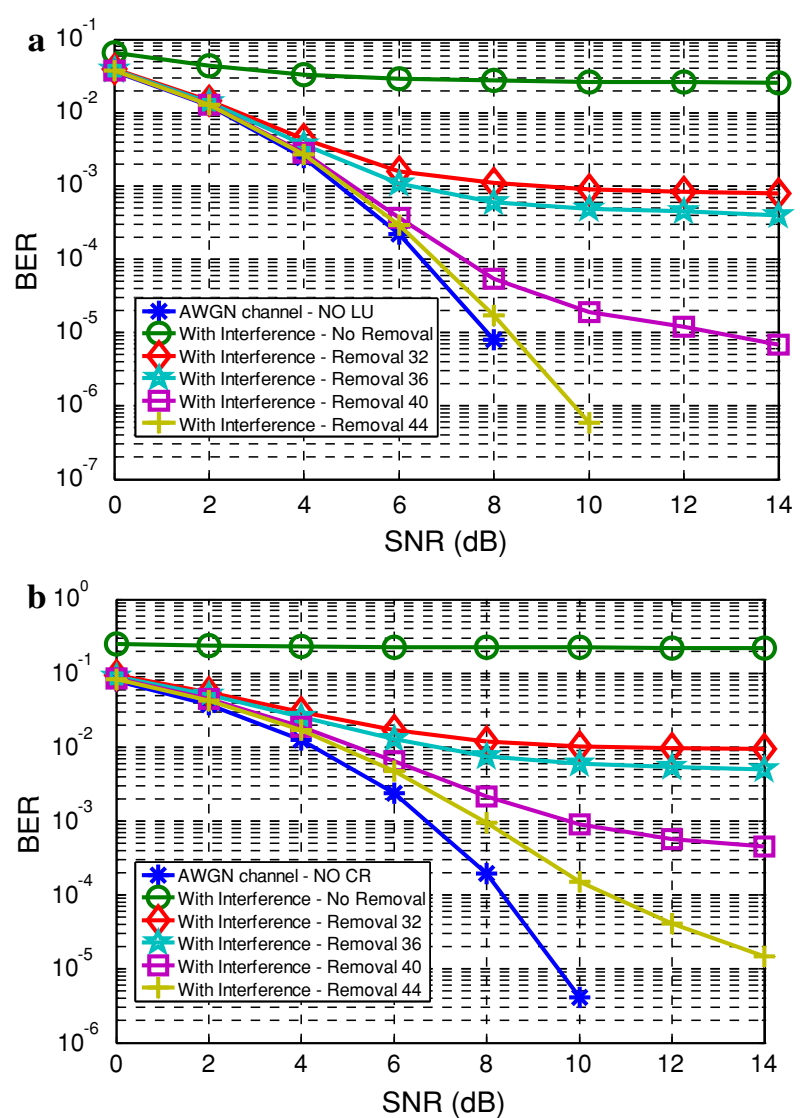

Fig. 9 a Performance of WP-MCM based CR system in the presence of LU, $\mathbf{b}$ BER performance of LU in the presence of the WP-MCM based CR system. The carriers of CR in the region of the LU are removed to enable it to co-exist with LU. The wavelet used is the maximally frequency selective filter bank with characteristics $L=50, K=19, B=0.1$

\subsubsection{Carrier Deactivation and System Performance}

Figure 10 depicts the effect of removal of carriers on the system performance. The SNR is fixed at $8 \mathrm{~dB}$. From the plots we may note that BER of RU and LU systems reduce by increasing the number deactivated carriers however, after reaching a specific number of deactivated carriers, further removal does not increase CR and LU system performance and that there is a saturation point beyond which removing anymore carriers has no benefit. For the setup considered the performance plateaus when 42 or more carriers are removed.

\subsection{Comparison of Wavelets-Performance Comparison of the System When Using} the Maximally Frequency Selective Filter Bank with Respect to Conventional Wavelets of Similar Lengths

Here we equate the performance improvements brought by maximally frequency selective filter bank with a few known wavelets. The wavelets considered are Daubechies-15, Coiflet-5 
Fig. 10 Number of carriers deactivated and their effect on system performance. The BER curves are plotted for a SNR of $8 \mathrm{~dB}$

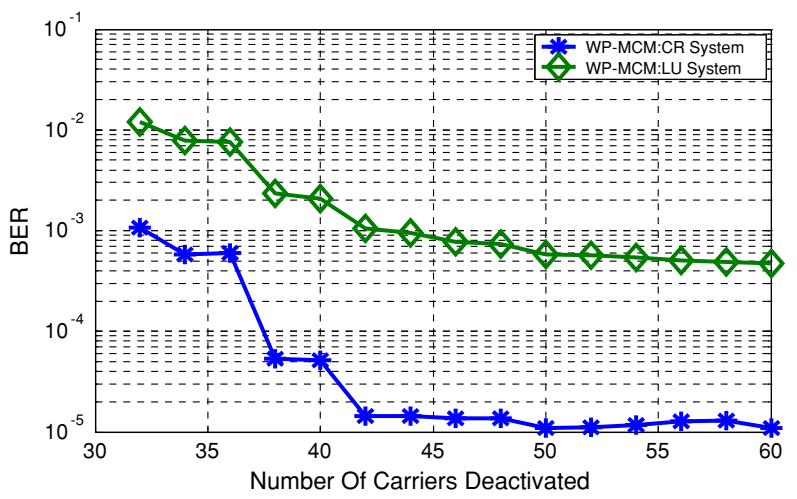

Fig. 11 a Frequency responses of the LPF-HPF pairs of various wavelets, $\mathbf{b}$ wavelet Packet bases as MCM carriers (only first four carriers shown). The wavelets considered are Daubechies-15, Symlet-15, Coiflet-5, Maximally Frequency Selective wavelet with $L=30, K=12, B=0.1$. The Daubechies-15 and Symlet-15 responses almost match and hence the responses of Symlet-15 are not visible
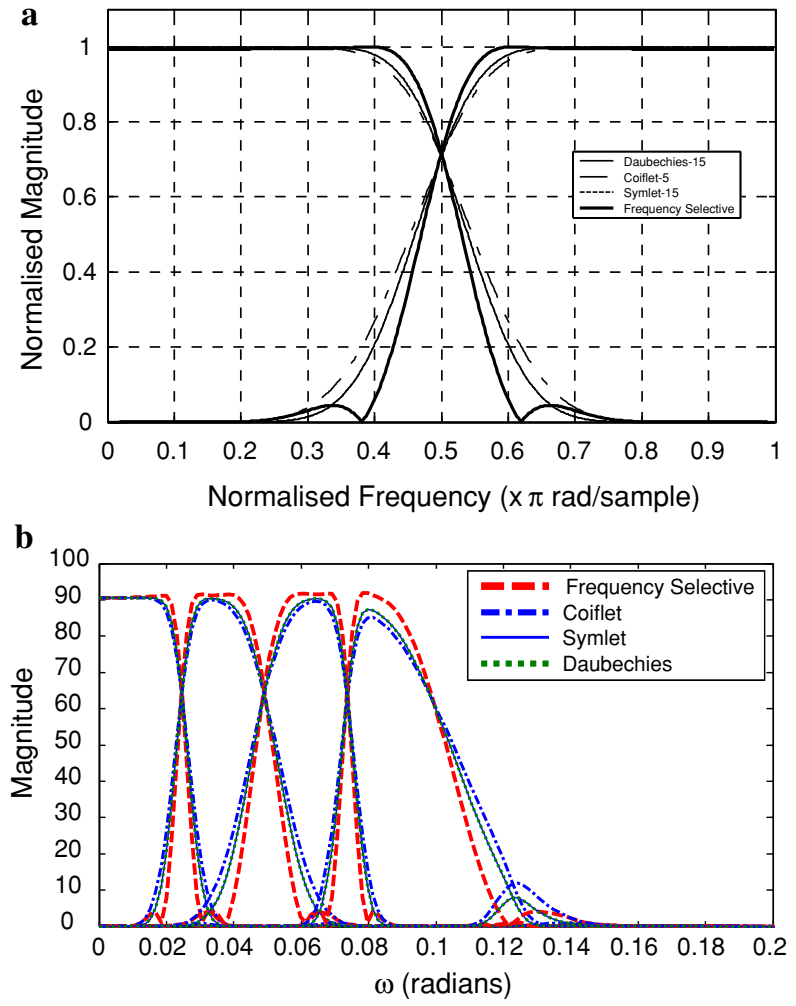

and Symlet-15. For the fairness of comparison, all the filter banks are taken to be of the same length $(L=30)$. The system performance has a direct correlation to the frequency selectivity of the filter bank used to derive the carriers. This effect is exemplified in Fig. 11a, where the frequency responses of the wavelet families has been plotted, and in Fig. 11b which shows the corresponding MCM carriers. The BER performance curves of the CR and LU systems have been plotted in Fig. 12a and b, respectively. The maximally frequency selective filter banks have the narrowest transition band and hence they clearly outperform the other wavelet families while Coiflet- 5 is the worst of the lot because of its very wide transition band. 
Fig. 12 Comparison of different wavelets: a BER performance of the WP-MCM based CR system in the presence of $\mathrm{LU}, \mathbf{b}$ BER performance of LU in the presence of the WP-MCM based $\mathrm{CR}$ system. The carriers of $\mathrm{CR}$ in the region of the LU are removed to enable it to co-exist with LU. FS denotes the maximally frequency selective wavelet, $\mathrm{db}$ denotes Daubechies, sym-symlet, coif stands for coiflet
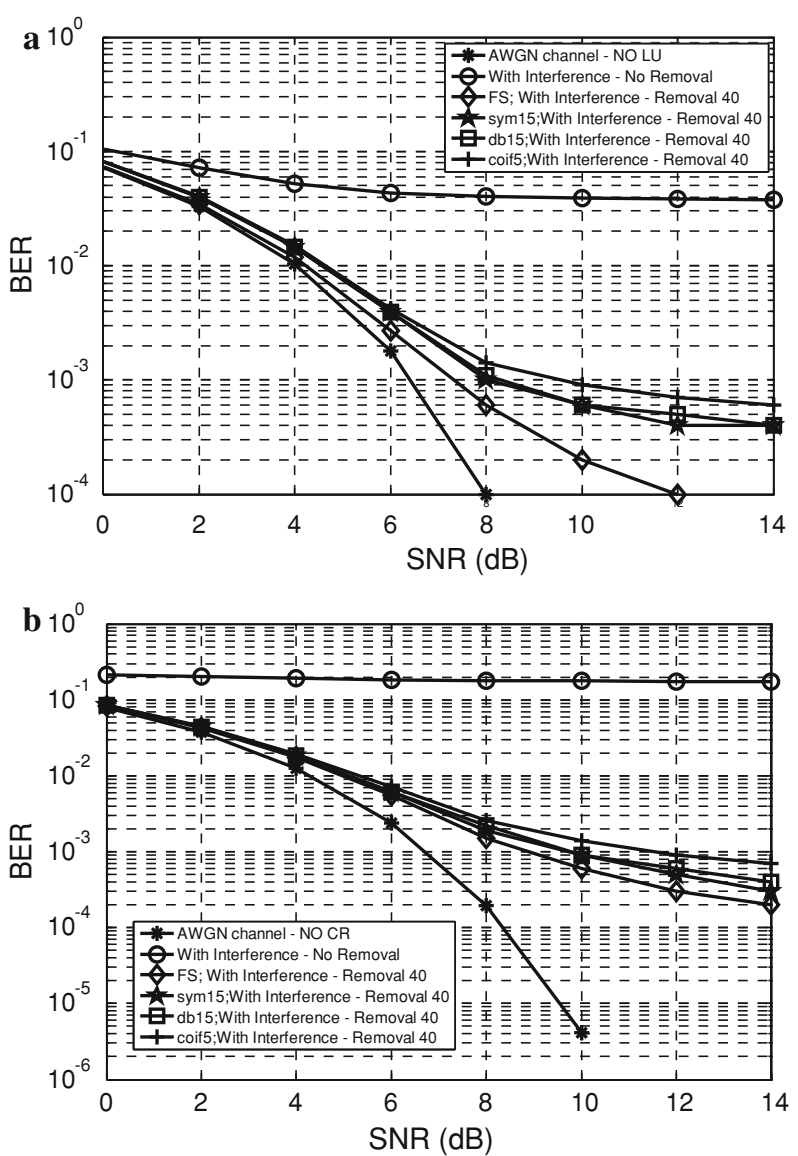

Further, Fig. 13 shows the comparison of the spectrum of different WP-MCM based CR system with carrier removals in the region of the LU. Frequency selective wavelets ensure a neat separation of the CR and LU footprints while all other wavelets have residual infringing components. To further corroborate this we may refer to Fig. 14 which depicts the effect of removal of carriers on the system performance. The SNR is fixed at $8 \mathrm{~dB}$. From the plots we may note that the usage of maximally frequency selective filters requires less carrier removals and gives a better SNR gain.

\subsection{Comparison of Performance of WP-MCM and OFDM Based CR Systems}

It will be interesting to see as to how the WP-MCM construction devised in this paper matches up to traditional OFDM implementations. For fairness of comparison both the OFDM and WP-MCM scheme use the same set of transmission parameters.

\subsubsection{Operation of $C R$ in the Presence of $L U$}

As illustrated in Fig. 15a, the performance of both the OFDM and WP-MCM based CR systems worsens (WP-MCM marginally more tolerant than OFDM) in the presence of LU 

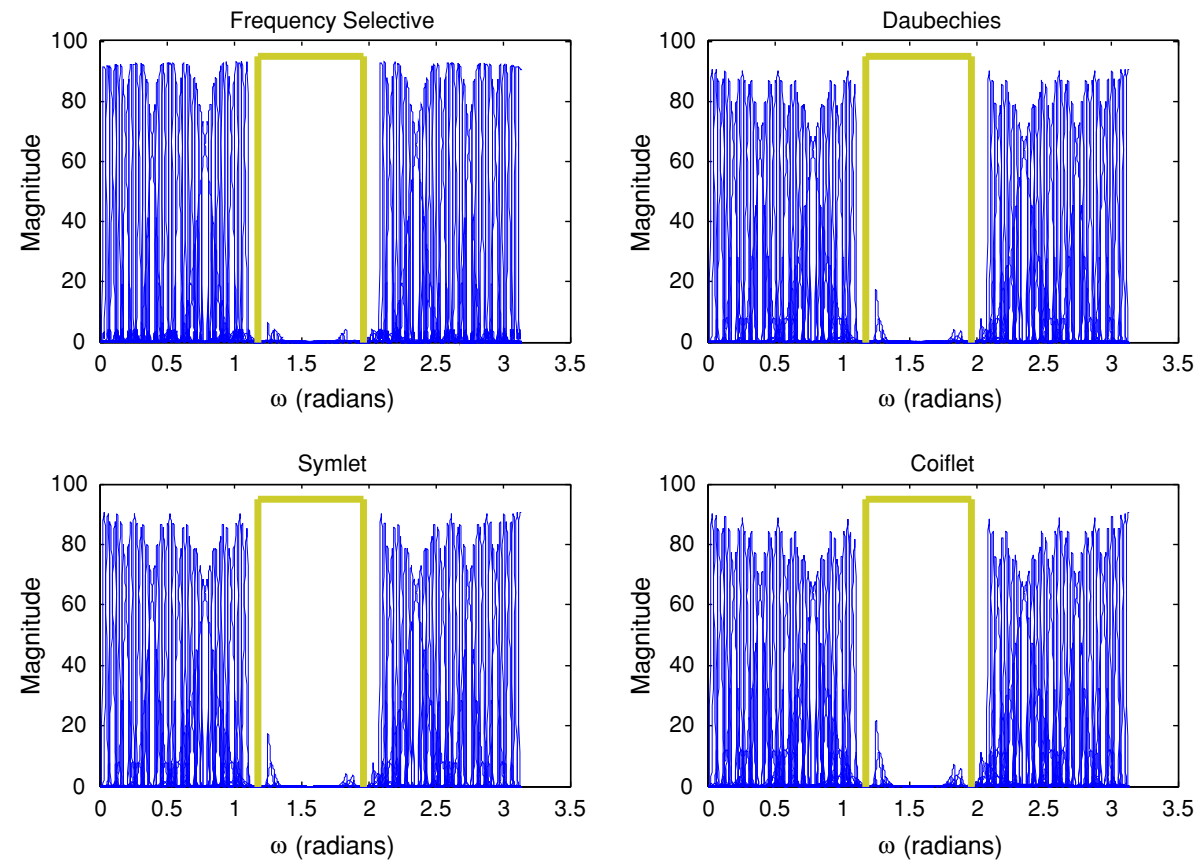

Fig. 13 Comparison of the spectrum of different WP-MCM based CR system with carrier removals in the region of the LU. The rectangle marks the LU spectral footprint

Fig. 14 BER performance of different wavelets: number of carriers deactivated and their effect on system performance. The BER curves are plotted for a SNR of $8 \mathrm{~dB}$. FS denotes the maximally frequency selective wavelet, db denotes Daubechies, sym-symlet, coif stands for coiflet

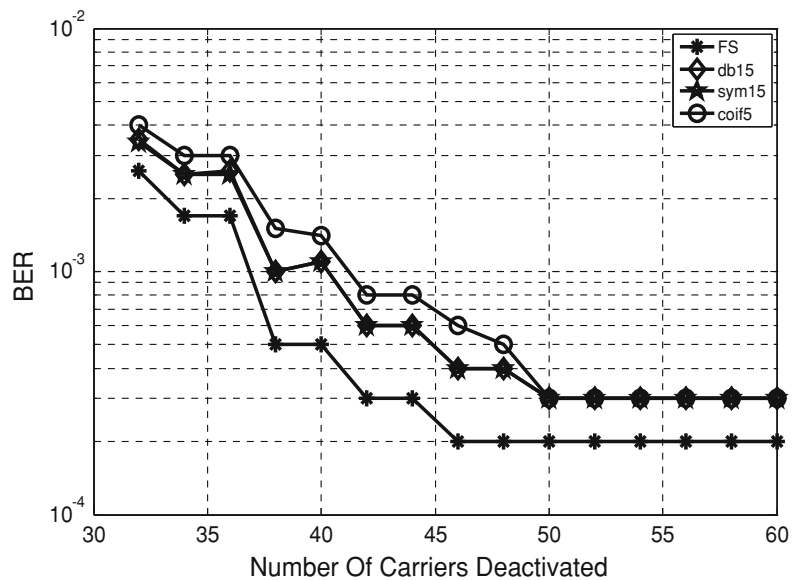

and perform better when their carriers in and around the LU spectral bands are removed. For OFDM the best results are obtained when 36 or more carriers are removed while for WP-MCM the number is about 40 carriers. The advantage of WP-MCM is that once its transmission signal is steered clear of the LU bands, it comfortably outperforms its OFDM counterpart. For e.g. for a given BER of $10^{-4}$ with 44 carrier removals, WP-MCM gives a gain of about $2 \mathrm{~dB}$ in comparison to OFDM. 

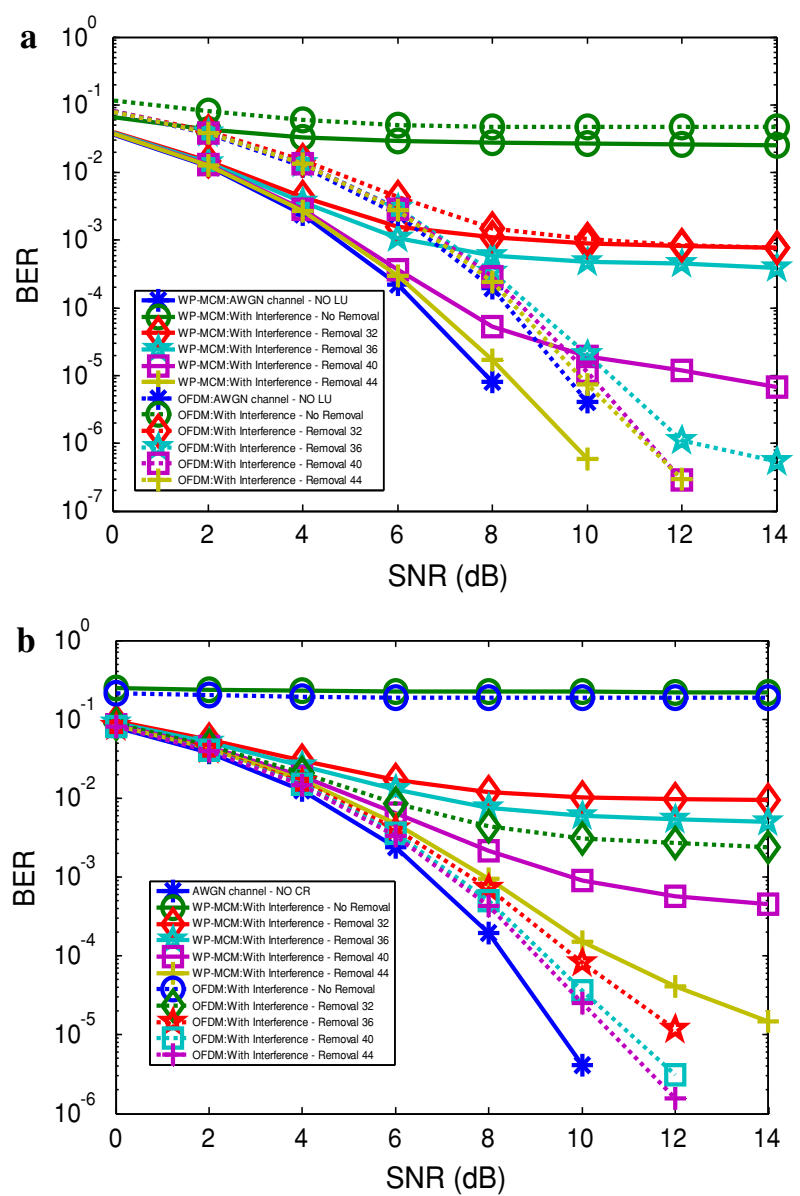

Fig. 15 OFDM versus WP-MCM based CR. a BER performance comparison of OFDM and WP-MCM based CR systems in the presence of LU, $\mathbf{b}$ influence of WP-MCM and OFDM based CR systems on the operation of LU

\subsubsection{Comparison of Performances of LU Under OFDM and Wavelet Packet Based MCM Respectively}

The LU performance worsens in the presence of both WP-MCM and OFDM CR systems and as in the previous case, removal of CR carriers brings relief (Fig. 15b). OFDM needs a lower number of carriers to be removed in comparison to WP-MCM to restore system performance to the levels of AWGN.

\subsubsection{Carrier Deactivation and System Performance}

Figure 16 depicts the effect of removal of carriers on the system performance. The SNR is fixed at $8 \mathrm{~dB}$. From the plots we may note that though OFDM requires less carrier removals, WP-MCM based CR gives a better SNR gain. Another point to note is that removing the carriers does promote cohabitation of the CR and LU systems but this has a saturation point beyond which removing anymore carriers has no benefit. 
Fig. 16 OFDM versus WP-MCM based CR: number of carriers deactivated and their effect on system performance. The BER curves are plotted for a SNR of $8 \mathrm{~dB}$

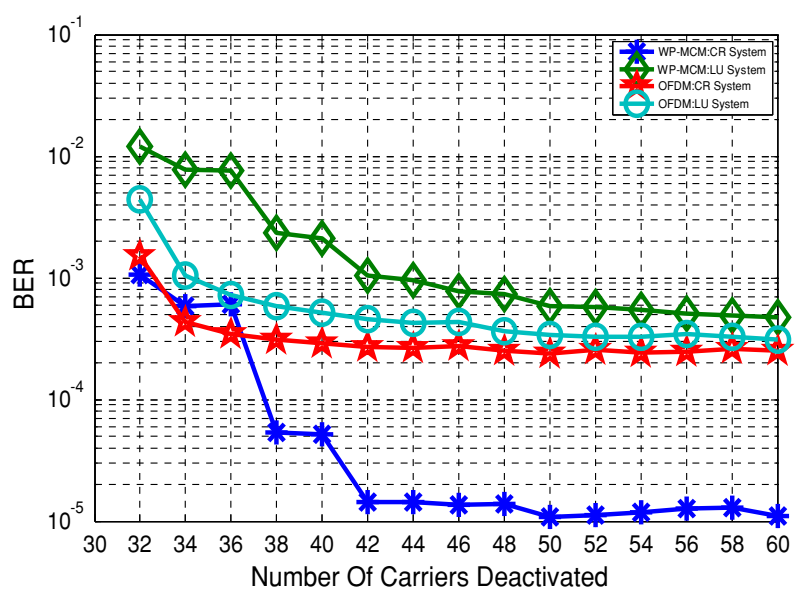

\subsection{Impact of Licensed User Channel Occupancy Time on Performance of WP-MCM Based CR Systems}

In all the simulations considered above the LU is taken to be transmitting simultaneously with the CR system with a $100 \%$ channel occupancy. It would be interesting to consider the situation where the LU has a channel occupancy characterized by a probability of transmission less than $100 \%$. Figure 17 shows the BER performance curves for the CR system when the LU operates with $100 \%, 50 \%, 25 \%$ and $12.5 \%$ channel occupancy. The CR configuration considered is with 44 of the WP-MCM carriers removed in and around the LU band.

\subsection{Mutual Interference When more than One CR or LU Strives to Occupy the Channel}

Finally we present results that seek to demonstrate the impact of mutual interference due to multiple CR or LU users. Fig. 18a and b, respectively, depict:

(a) the BER curves for the impact on CR performance with increasing number of LU-In this case a single CR configuration with 44 of the WP-MCM carriers removed in and

Fig. 17 LU channel occupancy impact on WP-MCM based CR. BER performance comparison of WP-MCM based CR systems in the presence of LU with different channel occupancy probabilities

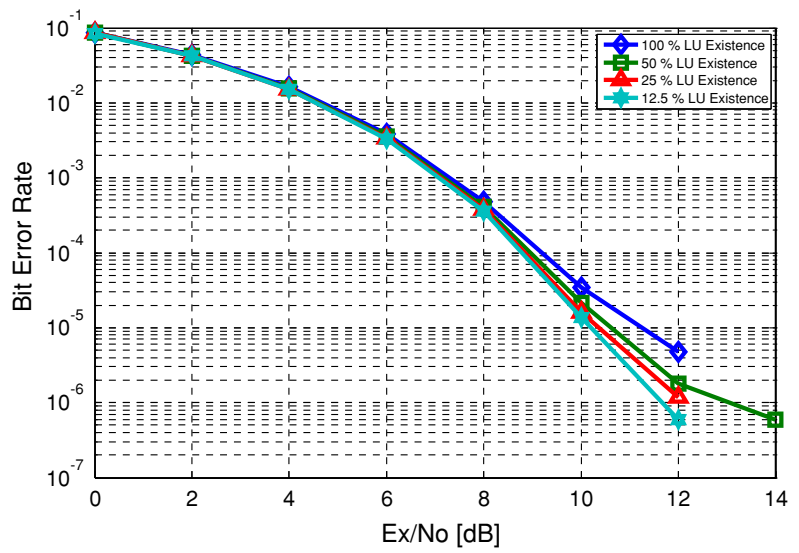



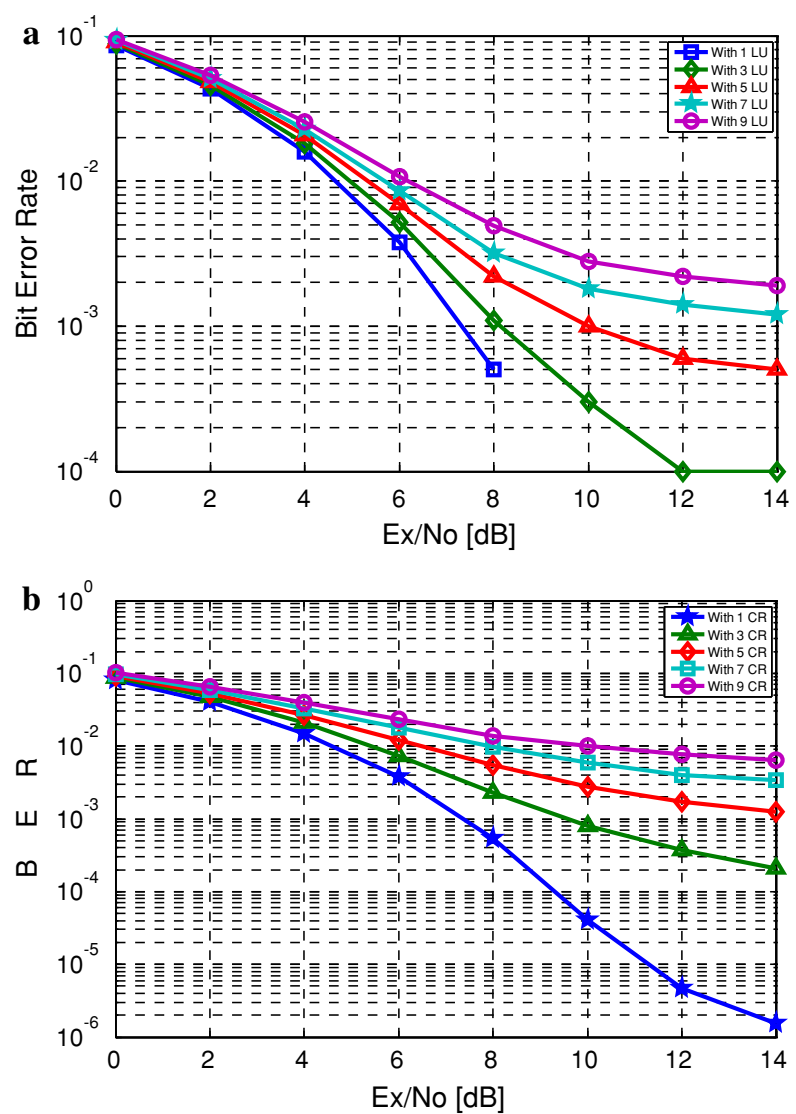

Fig. 18 OFDM versus WP-MCM based CR. BER performance comparison of OFDM and WP-MCM based CR systems in the presence of LU. a Impact on CR performance with increasing number of LU, $\mathbf{b}$ impact on LU performance with increasing number of $\mathrm{CR}$

around the LU band is considered. All the LUs are taken to be operating in the same band and with the same power.

(b) the BER curves for the impact on LU performance with increasing number of CR-In this case a single LU system with roughly 32 carriers is considered. All the CRs are taken to be operating in the same band and with the same power and with 44 of the WP-MCM carriers removed in and around the LU band.

\section{Concluding Remarks}

A novel wavelet packet based multi-carrier modulation scheme for use in Cognitive Radio systems was proposed. Cohabitation of the CR system with LU is actualized by dynamically activating/deactivating the CR carriers in a way that the CR and LU systems do not have any competing time-frequency components. The carriers of the WP-MCM system are generated by multistage tree-structured paraunitary filter banks. The emphasis was on deriving optimal maximally frequency selective wavelet packet bases that best suit applicability to 
spectrum shaping in CR systems. Wavelets including Daubechies-15, Coiflet-5 and Symlet15 families were used for the study. Through simulation studies the usefulness and potential of the WP-MCM based for developing CR systems was demonstrated. Comparing with traditional OFDM implementations, the performance of the proposed system in the presence of a LU matches quite well. Although the results in this effort were obtained in a relatively benign setup, we believe that the benefits and advantages gained by the system can be replicated even in complicated propagation environments and fading channels. Our next step is to broaden the study and include multipath fading channel and assess the performance achievement of the proposed system.

The real motivation for wavelets for cognitive radio lies in their flexibility and adaptation. Unlike conventional Fourier bases which are static in nature, wavelets offer multitude of variations which can be customised to the requirement in hand. In this article, the design criterion is set to avoid interference to licensed primary users. This can be easily furthered by altering the design mechanism to include other requirements such as reduction of ISI/ICI or Peak-to-average Power Ratio (PAPR).

While the benefits of the proposed system have been documented, it must be acknowledged that a few issues remain to be addressed before such schemes can become popular. Primary amongst them are the issues of system complexity and implementation. Moreover, questions on how the system designed under a chosen criterion would perform with respect to other criteria have to be addressed. For example, is it possible to design a system with minimum interference to LUs, but still have reasonable PAPR (or the way around)? And will the tradeoffs be reasonable? These issues have to be adequately covered.

As wireless devices are gradually evolving to become sophisticated, pervasive and intelligent rather than just fancy telecommunication gadgets, perhaps the stringent complexity constraints will be more relaxed. Moreover, the application of wavelet theory to telecommunication systems design is a very recent phenomenon and a work in progress. With advancements to mathematics of wavelet theory and some engineering ingenuity the bottlenecks in actualizing wavelet based systems can be overcome paving the way for successful implementation of the scheme.

In conclusion, the performance results of the simulation studies make us to conclude that the novel proposed system can be fruitfully used to construct adaptive wireless systems.

Open Access This article is distributed under the terms of the Creative Commons Attribution Noncommercial License which permits any noncommercial use, distribution, and reproduction in any medium, provided the original author(s) and source are credited.

\section{References}

1. Bouwel, C. V., Potemans, J., Schepers, S., Nauwelaers, B., \& Capelle, A. V. (2000, October). Wavelet packet based multicarrier modulation. In Proceedings of the IEEE benelux symposium on communications and vehicular technology. Leuven, Belgium.

2. Burke, B. (1998, May). The world according to wavelets: The story of a mathematical technique in the making (2nd ed.). A K Peters.

3. Burrus, C. S., Gopinath, R. A., \& Guo, H. (1998). Introduction to wavelets and wavelet transforms, a primer. Upper Saddle River, NJ: Prentice Hall.

4. Cohen, A., \& Kovacević, J. (1996, April). Wavelets: The mathematical background. Proceedings of the IEEE, 84(4), 514-522.

5. Daubechies, I. (1992). Ten lectures on wavelets. Philadelphia: SIAM.

6. Haykin, S. (2005, February). Cognitive radio: Brain-empowered wireless communications. IEEE Journal On Selected Areas In Communications, 23(2), 201-220. 
7. Jamin, A., \& Mahonen, P. (2005, March). Wavelet packet modulation for wireless communications. Wireless Communications \& Mobile Computing Journal, 5(2), 123-137.

8. Jensen, A., \& Cour-Harbo, A. L. (2001). Ripples in mathematics: The discrete wavelet transform. Springer.

9. Lakshmanan, M. K., \& Nikookar, H. (2006, May). A review of wavelets for digital wireless communication. Wireless Personal Communications, 37(3-4), 387-420.

10. Lakshmanan, M. K., \& Nikookar, H. (2007, June). Mitigation of interference from wideband IEEE 802.11a source on UWB wireless communication using frequency selective wavelet packets. In 3 rd international waveform diversity and design conference. Pisa, Italy.

11. Lakshmanan M. K., \& Nikookar, H. (2007, September). Wavelet packet based strategy to mitigate wideband interference on impulse radio. In 18th international symposium on personal, indoor and mobile radio communications (PIMRC), Athens, Greece.

12. Lindsey, A. (1997, May). Wavelet packet modulation for orthogonally transmultiplexed communications. IEEE Transaction On Signal Processing, 45, 1336-1339.

13. Mallat, S. (1989, July). A theory for multiresolution signal decomposition: The wavelet representation. IEEE Transactions on Pattern Analaysis and Machine Intelligence, 11, 674-693.

14. Mitola, J. III. (2000, May). Cognitive radio: An integrated agent architecture for software defined radio. Doctoral Dissertation, KTH, Stockhold.

15. Negash, B. G., \& Nikookar, H. (2000, October). Wavelet-based multicarrier transmission over multipath wireless channels.IEE Electronics Letters 36(21), 1787-1788.

16. Oppenheim, A. V., \& Schafer, R. W. (1989). Discrete-time signal processing. Englewood Cliffs, NJ: Prentice Hall.

17. Rioul, O., \& Duhamel, P. (1994, August). A Remez exchange algorithm for orthonormal wavelets. IEEE Transactions on Circuits Systems-II, 41(8), 550-560.

18. Rioul, O., \& Duhamel, P. Note on 'a Remez exchange algorithm for orthonormal wavelets'. Authors note available at http://www.comelec.enst.fr rioul/publis/199302rioulduhamelnote.pdf.

19. Strang, G., \& Nguyen, T. (1996). Wavelets and filter banks. Wellesley-Cambridge Press.

20. Vaidyanathan, P. P. (1993). Multirate systems and filter banks Englewood Cliffs, NJ: Prentice-Hall, Inc.

21. Vetterli, M., \& Kovacevic, I. (1995). Wavelets and subband coding. Englewood Cliffs, NJ: Prentice Hall PTR.

22. Weiss, T. A., \& Jondral, F. K. (2004, March). Spectrum pooling: An innovative strategy for the enhancement of spectrum efficiency. IEEE Communications Magazine, 42, S8-S14.

\section{Author Biographies}

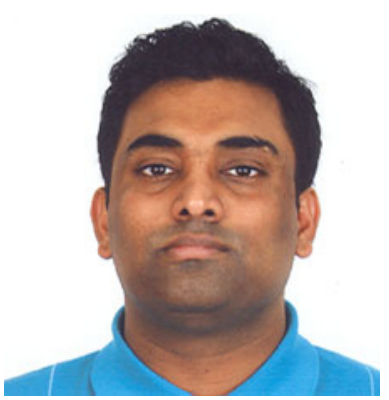

M. K. Lakshmanan received his the B.E. (with distinction) in electrical engineering from the University of Madras, Chennai, India, in 2000. In 2000, he joined the Indian Software firm, Polaris Software Labs Ltd., where he wrote software for Telecommunication applications. At Polaris, he was awarded the "On The Spot Of Excellence Award" for his efforts. In 2003, he moved to the Indian Institute of TechnologyMadras, India, to lead a team of junior researchers to develop and establish a wireless communications network for rural connectivity. In 2004, he was granted the Royal Dutch/Shell Chevning scholarship to pursue a Masters program in Telecommunications at the Delft University of Technology (TU Delft). He completed his Masters (with CUM LAUDE) in the 2006 and continued with his doctoral studies at TU Delft where he is currently conducting research in the fields of wavelets, signal processing and Wireless Communications at the International Research Center for Telecommunications Transmission and Radar (IRCTR). In December 2007 he won the BEST STUDENT PAPER award at The 10th International Symposium on Wireless Personal Multimedia Communications, Jaipur, India. 


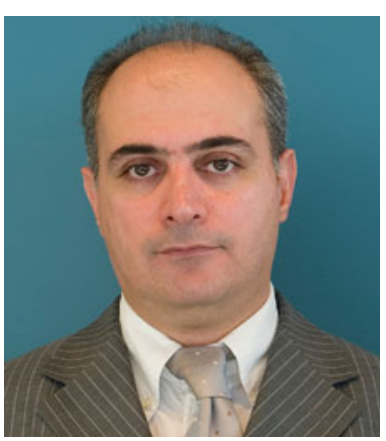

H. Nikookar received his Ph.D. in Electrical Engineering from Delft University of Technology (TUDelft), The Netherlands, in 1995. He is an Associate Professor at the International research Centre for Telecommunications and Radar (IRCTR) of the Department of Electrical Engineering, Mathematics and Computer Science of TUDelft. He is also the leader of the Radio Advanced Technologies and Systems (RATS) Program of IRCTR. Dr. Nikookar has conducted research in many aspects of wireless communications, including wireless channel modeling, UWB, MIMO, multicarrier transmission, Wavelet-based OFDM and Cognitive Radio. He is a senior member of the IEEE. 